\title{
Laser annealed low temperature deposited polysilicon waveguides for nonlinear photonics
}

\author{
Y. Franz ${ }^{1}$, A. F. J. Runge ${ }^{1}$, S. Z. Oo ${ }^{1,2}$, N. Healy ${ }^{1,3}$, G. Martinez-Jimenez ${ }^{1}$, A. Z. Khokhar ${ }^{1,2}$, A. Tarazona ${ }^{1}$, \\ H. M. H. Chong ${ }^{2}$, S. Mailis ${ }^{1}$, and A. C. Peacock ${ }^{1}$ \\ 1. Optoelectronics Research Centre, University of Southampton, Highfield, Southampton, SO17 1BJ, U. K. \\ 2. School of Electronics and Computer Science, University of Southampton, Highfield, Southampton, SO17 1BJ, U. K. \\ 3. Emerging Technology and Materials Group, Newcastle University, Merz Court, Newcastle, NE1 7RU, U.K.
}

The intrinsic properties of silicon $(\mathrm{Si})$ make it an excellent material for integrated photonics devices with small footprints [1]. To date, most of the reported devices have been based on crystalline silicon (c-Si), but this material suffers from difficult integration with electronic layers due to fabrication constraints. Subsequently, there has been growing interest in alternative forms of $\mathrm{Si}$, such as hydrogenated amorphous silicon (a-Si:H), silicon nitride ( $\mathrm{SiN}$ ) and polysilicon (poly-Si) [2]. Among these materials, only poly-Si has the potential to exhibit both optical and electronic properties that are equivalent to c-Si. However, to achieve good material quality, poly-Si is typically deposited at temperatures higher than $900^{\circ} \mathrm{C}$, rendering it incompatible with most CMOS fabrication processes. Thus there is a growing drive to develop new techniques to deposit poly-Si at low temperatures $\left(<450^{\circ} \mathrm{C}\right)$ [2], but so far the optical losses in these materials have been high, limiting its use in all-optical systems employing nonlinear optical processing. In this work, we report the fabrication and characterisation of laser annealed, low temperature deposited silicon waveguides with low optical losses. Our results represent the first demonstration of nonlinear propagation in a poly-Si waveguide suitable for integration.

The waveguide fabrication process starts with the deposition of $400 \mathrm{~nm}$ of amorphous silicon (a-Si) on a $4.7 \mu \mathrm{m}$ thick silica box layer using a hot-wire chemical vapor deposition technique, at a temperature of $320^{\circ} \mathrm{C}$ [3]. Then, using e-beam lithography and dry etching, $2 \mu \mathrm{m}$ wide wires are patterned on the a-Si film. Next, the pre-patterned waveguides are crystallised using a continuous wave argon ion laser emitting at $488 \mathrm{~nm}$. By adjusting the power and scanning speed of the laser, thermal energy is transferred to the a-Si material, which melts and crystallises, forming large crystal grains along the microstructure. The processed waveguides display an ultra-smooth top surface, as shown in Fig. 1(a), while x-ray diffraction measurements reveal single crystal-like properties over lengths greater than $40 \mu \mathrm{m}$. Using a cut back method, we measure linear optical loss as low as $5.13 \mathrm{~dB} / \mathrm{cm}$, suitable for the observation of nonlinear dynamics.

To assess the nonlinear properties of these waveguides we performed a set of measurements using a passively mode-locked laser emitting $750 \mathrm{fs}$ pulses centred at $1540 \mathrm{~nm}$ at a repetition rate of $40 \mathrm{MHz}$. First, we measured the power at the waveguide output for different input pulse powers. The results are shown in Fig. 1(b), where we can see a clear saturation of the output power due to the nonlinear absorption of the Si material originating from the two-photon absorption [4]. Next, we recorded the optical output spectra for different input powers using an optical spectrum analyser. The recorded spectra, shown in Fig. 1(c), display clear evidence of self-phase modulation induced spectral broadening when the input pulse power increases. Note that the sharp features modulating the spectral shape of the pulses arise from multimode interactions, due to the micron-sized width of the investigated waveguide [5]. However, as the laser processing procedure is highly confined, this method is also suitable for the fabrication of single mode nanoscale devices. Thus, this method opens a route for the development of low cost integrated nonlinear photonic systems based on poly-Si materials that are compatible with CMOS infrastructures. We thank the Diamond Light Source for access to the I18 beamline.
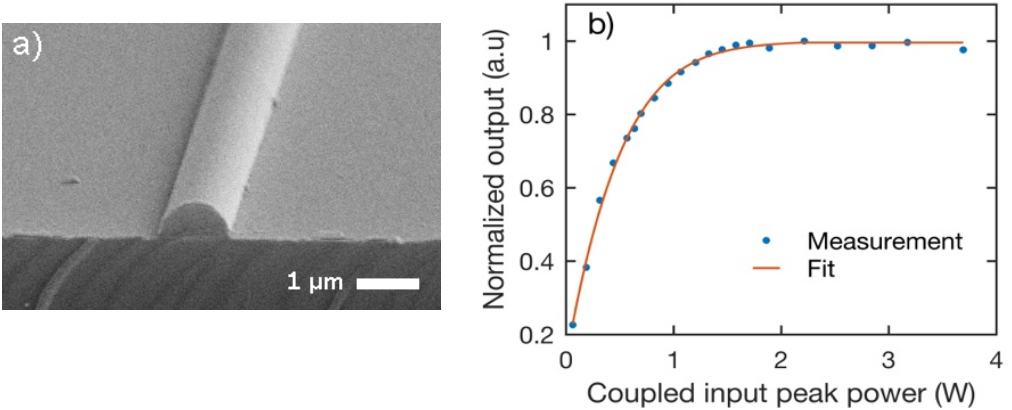

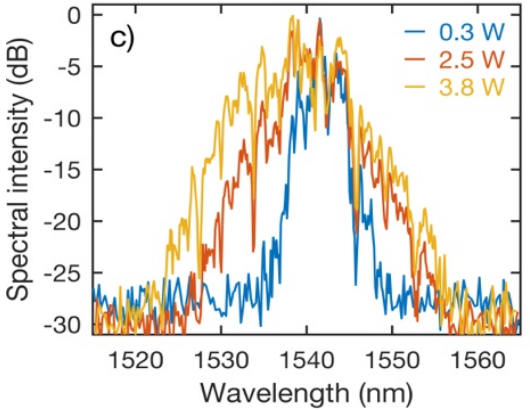

Fig. 1 (a) SEM image of a laser processed waveguide, (b) normalized output power as a function of the coupled input peak power, (c) output spectra for three different input pulse peak powers.

\section{References}

[1] V. R. Almeida, et al., "All-optical control of light on a silicon chip" Nature 431, 1081-1084 (2004).

[2] Y. H. D. Lee, and M. Lipson, “Back-End Deposited Silicon Photonics for Monolithic integration on CMOS,” IEEE J. Sel. Top. Quant. Electron. 19, 8200207 (2013).

[3] T. M. Ben Masaud, et al., "Hot-wire poly-Silicon waveguide with low deposition temperature," Opt. Lett. 38, 4030-4032 (2013).

[4] A. R. Motamedi, et al., "Ultrafast nonlinear optical studies of silicon nanowaveguides," Opt. Express 20, 4085-4101 (2012).

[5] A. C. Peacock, et al., "Nonlinear pulse dynamics in multimode silicon core optical fibers" Opt. Lett. 37, 3351-3353 (2012). 